\title{
Steady shocks around black holes produced by sub-keplerian flows with negative energy
}

\author{
D. Molteni ${ }^{1 \star}$, G. Gerardi ${ }^{1}$ and V. Teresi ${ }^{1}$ \\ ○ ${ }^{1}$ Dipartimento di Fisica e Tecnologie Relative, Università di Palermo, Viale delle Scienze, Palermo, 90128, Italy
}

\begin{abstract}
We discuss a special case of formation of axisymmetric shocks in the accretion flow of ideal gas onto a Schwarzschild black hole: when the total energy of the flow is negative. The result of our analysis enlarges the parameter space for which these steady shocks are exhibited in the accretion of gas rotating around relativistic stellar objects. Since keplerian disks have negative total energy, we guess that, in this energy range, the production of the shock phenomenon might be easier than in the case of positive energy. So our outcome reinforces the view that sub-keplerian flows of matter may significantly affect the physics of the high energy radiation emission from black hole candidates. We give a simple procedure to obtain analytically the position of the shocks. The comparison of the analytical results with the data of $1 \mathrm{D}$ and $2 \mathrm{D}$ axisymmetric numerical simulations confirms that the shocks form and are stable.
\end{abstract}

Key words: accretion, accretion disks — black hole physics — hydrodynamics instabilities

\section{INTRODUCTION}

The presence of shocks in accretion flows is a relevant fact in the theory of accretion discs. Shocks are a very efficient mechanism to produce high energy emission of radiation and to induce strong acceleration of particles. Spiral shocks in accretion disks in binary systems have been studied since long time, theoretically by Spruit (Spruit 1987), Chakrabarti (Chakrabarti 1990) and with both 2D and 3D numerical simulations (Sawada et al. 1987; Taam et al. 1991; Blondin et al. 1995; Yukawa et al. 1997). Furthermore there is increasing observational evidence of their significant role in determining the accretion of gas in binary systems (Hachisu et al. 2004). However the strength of this kind of shocks is usually weak.

A different scenario for shock production has been proposed by Chakrabarti (Chakrabarti 1990). It has been widely tested with numerical simulations (Chakrabarti \& Molteni 1993; Molteni et al. 1994; Rvu et al. 1996). In this view, an axisymmetric flow of gas, rotating with an angular velocity less than keplerian (we will call it "sub-keplerian" flow), and with small viscosity, can produce a steady shock. This fact is mainly due to the deceleration of the radial fall and consequent heating of the gas close to the centrifugal barrier. Although the origin of such flows seems unclear there are arguments to support the

\footnotetext{
* E-mail: molteni@difter.unipa.it (DM)
}

hypothesis that they really exist. In galactic nuclei the ambient gas feeding the central black hole may be quite well symmetric and with small rotation as shown by observations (Cohen \& Rvzhov 1997). On the theoretical side, taking into account also the role of the viscosity, a detailed study of the requirements for their origin from a keplerian disk has been produced (Chakrabarti 1996). These flows may explain very well the two frequencies in the QPO phenomenon in galactic black hole candidates (Chakrabarti et al. 2004). So even if their direct proof of existence is still pending, we think it is useful to study their properties and characteristics.

In any case it seems to us that the phenomenon, despite of its basic physical straightforwardness, has not been adequately considered. Maybe some "unconscious" questions are raised against their effective existence: 1) These shocks appear when the flow has well defined parameters, derived from the analytical treatment. 2) In the case of non ideal gas (i.e. if a real cooling, an heating or conduction are included) the analytical treatment is very difficult. 3) Even in the simple ideal gas case, the standard procedure (Chakrabarti 1990) to derive a solution is rather complicated: one has to compute the sonic point location and then compute the boundary conditions on derivatives of the radial speed, sound speed etc. at the sonic point itself, leading to cumbersome formulae. 4) At the end of the whole process the analytical solution is numerical and specific for the set of the chosen parameters. So no simple general formula, like the Shakura- Sunyaev disk structure formulae, is available. 
Another doubt is that the parameter space seems to be limited or too compelling. Roughly speaking, people might say that the sources of gas do not know the boundary conditions at the sonic point or at infinity, they spread out their gas as they like. So, for example, in bound systems, the total energy will be negative and, up to now, only positive energy solutions have been discussed and simulated. Even standard ADAF models (see Blandford \& Begelman (1999) and references therein), that are similar to the mentioned sub-keplerian flows, require positive energies to fit observational data. So what about negative energy flows? Here, we explain that, even in this case, there are solutions admitting permanent shocks. Obviously this does not mean that in every sub-keplerian flow there is a standing shock at work. But when the physical parameters of the accretion (essentially the rotation amount and the thermal energy content) are appropriate, then the shock process occurs and produces the relevant phenomena described in previous references.

The arguments of the paper are given as follows: in section 2 we revise the general accretion formulae for ideal gas with negative energy, in Section 3 we present the results of numerical simulations and in Section 4 we make our final discussion.

\section{ACCRETION OF IDEAL GAS WITH NEGATIVE ENERGY}

To explain the shock formation, let us resume the very basic physical ingredients of the phenomenon. We assume that an axisymmetric flow of an inviscid gas is falling from very large distance onto a black hole and has reached a steady state regime. We will adopt the Paczyński \& Wiita potential (Paczvński \& Wiita 1980) to mimic the general relativistic physics. The basic physical effect can be easily understood in terms of "classical" physics and it is well known that the Paczyński \& Wiita force reproduces many relativistic effects with high accuracy. As we said in the introduction, to obtain the steady state solution we may integrate the differential equations for mass, momentum and energy conservations. In this case one has to start the space integration from the sonic point as explained in the work by Chakrabarti (1990). However in the case of inviscid flow it is easy to find an algebraic implicit solution, since (in this case) the total energy is conserved. The Bernoulli theorem is valid and it can be exploited to close the system of equations and to find the solutions. For the $2 \mathrm{D}$ case (with real $\mathrm{Z}$ extension of the disk) we assume that the gas falls down in a condition of vertical equilibrium, with essentially zero vertical speed.

The mass conservation equation is given by:

$\dot{M}=-4 \pi r H \rho v_{r}=$ const

where $\mathrm{H}$ is the half thickness of the disk.

The vertical equilibrium hypothesis gives the well known expression for the half disk thickness:

$H=\frac{\sqrt{G M_{*} r}\left(r-r_{g}\right) a}{G M_{*}}$

We could use a more accurate formula, but the results are essentially unchanged while the formulae are more complicated.

According to the Bernoulli theorem the energy equation is given by:

$\frac{\lambda^{2}}{2 r^{2}}+\frac{1}{2} v_{r}^{2}+\epsilon(r)+\frac{P(r)}{\rho(r)}+\Psi(r)=E$

We will look for isentropic solutions and then we will see how to connect solutions with different entropies by shocks. For a flow with constant entropy $S$ and with specific heat at constant volume $C_{v}$ we may exploit the adiabatic relation:

$\rho=e^{-\frac{S}{C_{v}(\gamma-1)}}\left(\frac{a^{2}}{\gamma}\right)^{\frac{1}{(\gamma-1)}}$

that allows us to eliminate the density $\rho$ from the equations and to use the sound speed.

So we have two unknown quantities $v_{r}, a$ and two equations. Resolving for $a$ from the Bernoulli relation, using the radial Mach number $M=-\frac{v_{r}}{a}$, and putting all terms into the continuity equation, we have the following implicit solution for the Mach number:

$\dot{M}=-4 \pi A(r) f(M)$

where $f(M)$ is the Mach function which depends only on the $M$ values, given by:

$$
\begin{aligned}
& f(M)=q \frac{M}{\left[(\gamma-1) M^{2}+2\right]^{\frac{\gamma}{\gamma-1}}} \\
& \text { where } q=(\gamma-1)^{(\gamma-1)^{-1}} .
\end{aligned}
$$

This function (we name it the "Mach" function) has a maximum at $M_{\max }$, whose value is $M_{\max }=\frac{2}{\sqrt{2(\gamma+1)}}$

$A$ is function only of $r$; it is given by:

$$
\begin{gathered}
A(r)=w r^{-\frac{3+\gamma}{2(\gamma-1)}}\left[r^{2}\left(E-1 / 2 \frac{L^{2}}{r^{2}}+\frac{G M}{r-r_{g}}\right)\right]^{\frac{\gamma}{\gamma-1}}\left(r-r_{g}\right) \\
\text { with } w=2^{\frac{\gamma}{\gamma-1}} \gamma^{-(\gamma-1)^{-1}}(\gamma-1) e^{-\frac{S}{C v(\gamma-1)}} \frac{1}{\sqrt{G M}} .
\end{gathered}
$$

If the $\mathrm{E}$ value is positive this $A(r)$ function may have two minima. The inner minimum appears when the $\lambda$ value increases. When the total energy of the gas is negative we expect the system to be bound and therefore the solutions describing gas inflow into the black hole should not extend to infinity. Now the $A(r)$ function goes asymptotically to zero at a maximum radius and may have a relative minimum, at the radial position $r_{1}$, close to the black hole if the angular momentum is large enough. Figure 1 shows the A function for $\lambda=1.67$ and different $\mathrm{E}$ values $(-0.00001,-0.00003$, $0.00009,-0.00027)$; higher lines correspond to larger $E$ values (smaller absolute values). Figure 2 shows the A function for the same energy $E=-0.00001$ and different, increasing, angular momentum values $(1.67,1.67 \times 1.01,1.67 \times 1.02$, $1.67 \times 1.03)$; higher lines correspond to lower $\lambda$ values. Both figures are drawn with $S=0$ and $r$ starting from $r=1.5$ to avoid the singularity at $r=1$. For the same $S$ value and with $\gamma=\frac{4}{3}$ we have the value $w=3.181980514$.

The radius $r_{0}$ at which $A(r)$ goes to zero can be found analytically; it depends only on $\mathrm{E}$ and $\lambda$, not on the $\gamma$ of the gas.

Since the product of the Mach function $f(M)$ and the $A(r)$ function must be constant along the flow and since the Mach function has a single maximum, then the minimum of $A(r)$ at $r_{1}$ must be coincident with the maximum of $f(M)$. 


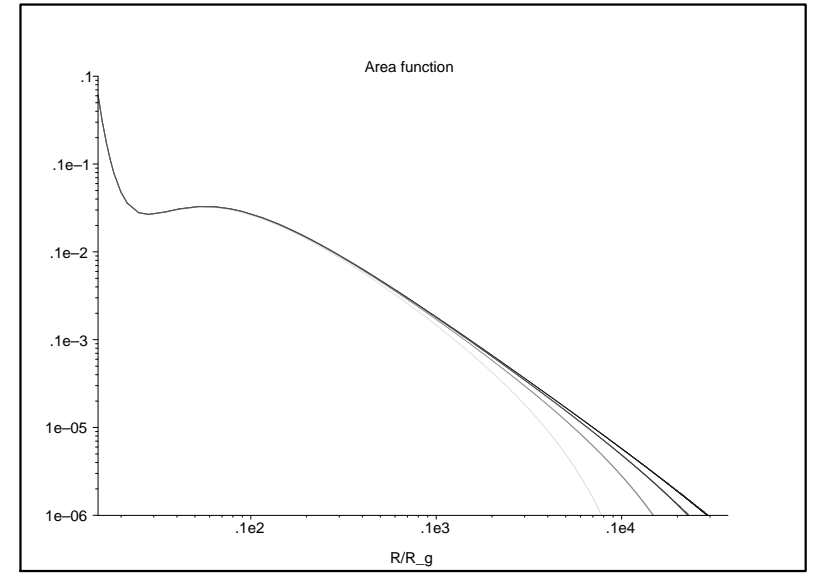

Figure 1. A(r) function for different E values. Higher lines correspond to larger $E$ values (smaller absolute values).

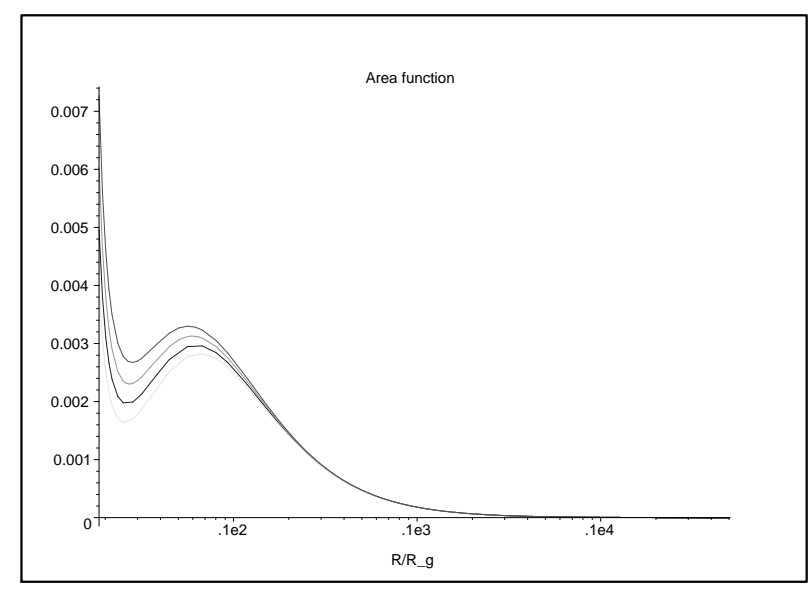

Figure 2. A(r) function for different angular momentum values. Higher lines correspond to lower $\lambda$ values.

Imposing $f(M) A(r)=f\left(M_{\max }\right) A\left(r_{1}\right)$ we obtain the solutions for $M$. Starting from the value $f\left(M_{\max }\right) A\left(r_{1}\right)$ we may obtain a new variety of solutions just solving for $\mathrm{M}$ the implicit equation $f(M) A(r)=f\left(M_{\max }\right) A\left(r_{1}\right) \xi$. The factor $\xi$ scales the entropy of the flow. Decreasing the value of $\xi<1$ we obtain families of solutions with the Mach number $M$ shifted to larger values.

For any supersonic solution $M_{\text {sup }}$, by using the Hugoniot relation we may calculate the post shock Mach value if a shock at a generic radius value $r$ occurred:

$M_{\text {post }}=\sqrt{\left(M_{\text {sup }}^{2}+2(\gamma-1)^{-1}\right)\left(2 \frac{M_{\text {sup }}^{2} \gamma}{\gamma-1}-1\right)^{-1}}$

A standing shock can occur in the solution at $r_{\text {shock }}$ if the values $M_{\text {post }}\left(r_{\text {shock }}\right)$ and $M_{\text {sub }}\left(r_{\text {shock }}\right)$ are equal:

$M_{\text {sub }}\left(r_{\text {shock }}\right)=M_{\text {post }}\left(r_{\text {shock }}\right)$

Among the solutions with different $\xi$ values (i.e. with different entropy values) we will find the ones that can be connected by a shock jump with the subsonic branch. Figure 3 shows the solutions for the Mach number with $\gamma=4 / 3$, $\lambda=1.67$ and $E=-0.00001$. The inner curve corresponds to $\xi=1$, the larger one to $\xi=0.5$. It is clear that the solutions

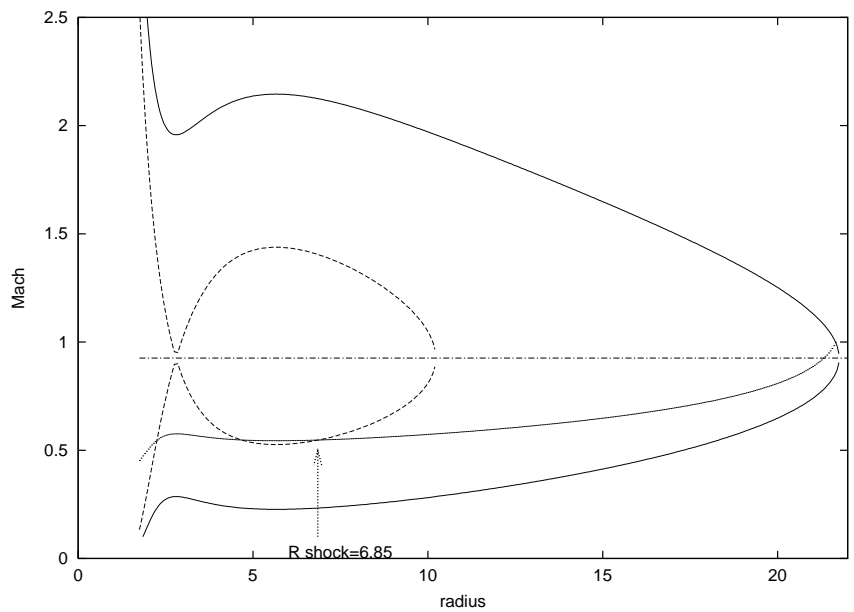

Figure 3. Analytical solution with shock. Mach number versus radius.

can reach a maximum radius $r_{m}=21.75$ : they do not extend to infinity. For this case the shock is at $r_{\text {shock }}=6.85$.

\section{NUMERICAL SIMULATIONS}

We report here the results of simulations performed using a Smoothed Particles Hydrodynamic (SPH) code in cylindrical coordinates developed by the authors. The SPH formulae have been reported in previous publications (Chakrabarti \& Molteni 1993). We stress here that a very accurate conservation of the angular momentum of the flow is an essential condition to obtain the shock solutions. For the numerical simulations we use dimensionless quantities. We adopt the speed of light as reference speed and the gravitational radius of a Schwarzschild black hole $r_{g}=2 G M_{*} / c^{2}$ as reference length.

The comparison of simulation data with $2 \mathrm{D}$ analytical solutions requires some care. In the analytical treatment we assumed vertical equilibrium, but numerical simulations allow the gas motion in the vertical direction. Therefore it is quite reasonable that the shocks, in the simulations, will not be exactly coincident with the theoretical predicted positions. We add, as comment, that we made simulations also with a fictitious damping acting only on the $v_{z}$ speed. The vertically damped simulations show a shock position in better agreement with theory.

We present here the data coming from the simulation of a $2 \mathrm{D}$ case with the parameters used to obtain Figure 3. This case has the following parameters: $E=-0.00001$, $\lambda=1.67$. The shock is predicted to be at $r_{\text {shock }}=6.85$. We chose to inject matter at $r_{i n}=20$. With this parameters the radial speed and the sound speed at $r_{i n}$ are $v_{i n}=-0.09606$, $a_{i n}=0.07790$ respectively. The spatial resolution of the $\mathrm{SPH}$ particle is $h=0.2$. The steady state particle number is $N_{p}=63000$.

Figure 4 shows the particle distribution in the upper half of the domain. The shock of the simulation is at $r_{\text {shock-SPH }}=8.7$.

Taking into account our previous remarks we may say that the shock position of the $2 \mathrm{D}$ simulation is in good 


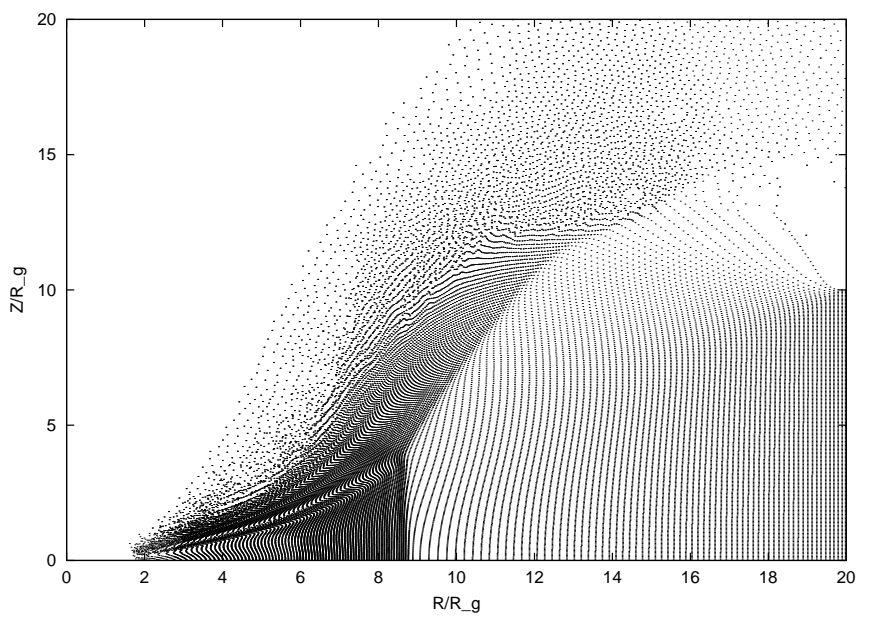

Figure 4. 2D simulation: Particle positions.

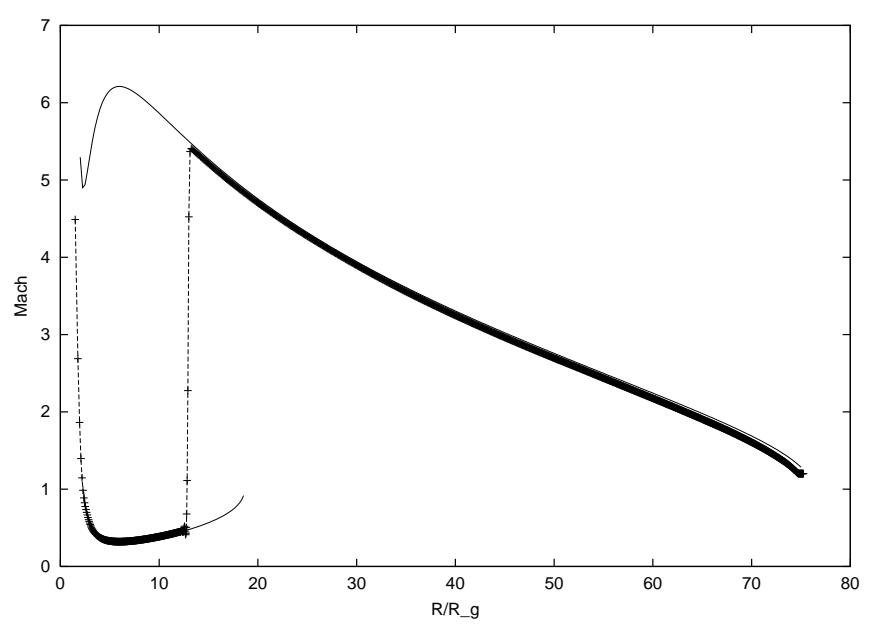

Figure 5. 1D simulation: Mach versus radial distance. The continuous line is the theoretical one.

agreement with the theory. We also report in Figure 5 the Mach value versus the radial distance of a $1 \mathrm{D}$ simulation. The physical parameters for the $1 \mathrm{D}$ simulation are $E=-0.003, \lambda=1.85, \gamma=5 / 3$; the numerical parameters are $r_{i n}=75.26, v_{i n}=-0.04719, a_{i n}=0.03931, h=0.125$. In this case, since there is no vertical motion, the agreement between theory and simulation must be exact, and effectively this occurs. For clarity, in the figure we plotted the theoretical Mach value plus a small shift(0.05). The shock is at the predicted position of $r_{\text {shock }}=12.8$. The whole shape of the curve profile is very well reproduced. Note that the $1 \mathrm{D}$ solution is obtained in the same way of the $2 \mathrm{D}$ case, but the functions $A(r)$ and $f(M)$ are different. (Molteni et al. 1999).

\section{CONCLUSIONS}

With our analysis we reinforce the view that the existence of permanent shocks around black holes is an important aspect of the physics of accretion flows. It has been sug- gested that the QPO oscillations of the X-ray luminosity of galactic black hole candidates may arise from shock oscillations (Chakrabarti et al. 2004).Jets of out-flowing matter may arise also from the high energetic post shock gas driven by radiation (Chattopadhvav et al. 2004). These shocks produce around the central object a bulge of hot gas comptonizing the low energy thermal emission from a cold keplerian disk. We remark that shocks produce the hot corona in a quite natural way, while the corona outcome or the ADAF solutions originating from keplerian disks have been shown by Molteni et al. to be very unlikely (Molteni et al. 2001).

We stress that these flows are essentially axisymmetric. So this situation is different from the one in which the gas flow, in a binary system, is captured from the wind originated by the normal star. Steady shocks of this kind cannot be produced in this case since the flow has low angular momentum, but has non-axisymmetric configuration.

Obviously a crucial point is the question of the origin of such symmetric sub-keplerian flows. It seems to us that this problem is not critical for black holes in the centers of galaxies or AGN, since in this case the star environment is nearly spherically symmetric and with low global rotation (Cohen \& Rvzhov 1997). In the case of black holes in binary systems we suggest that accretion may occur not only from the inner lagrangian point, but also by a small amount of ambient gas, possessing low rotation, in the potential well of the binary. Time dependent $3 \mathrm{D}$ numerical simulations (Molteni et al. 2001) and recent observations support this hypothesis, see Smith et al. (2002). Furthermore it was suggested by Carrol (Carrol D. L. 1990) that the PoyntingRoberston effect can be efficient also to drive matter from keplerian disks even in accretion on White Dwarfs. In the case of accretion on weakly magnetized neutron stars it has been shown (Miller \& Lamb 1993) that if the luminosity of the star is $\sim 0.2 L_{E}$ ( $L_{E}$ is the Eddington luminosity) a substantial fraction of the accreting matter can lose its angular momentum. We also think that, for the binary black hole case, the disk self-illumination may produce similar effect decreasing the angular momentum of the outer layers of the disks. This process would be similar to the accretion driven by radiation suggested by Ballantyne (Ballantvne \& Everett 2005) to occur in neutron star luminosity bursts. We add a trivial consideration: even if the inflow parameters do not correspond to the exact analytical ones, but are close to them, steady shocks may be still formed.

If requested, the authors can offer the software to reproduce both the analytical and the numerical results.

\section{REFERENCES}

Ballantyne, D. R., Everett, J. E., 2005, ApJ, 626, 364-372

Blandford, R. D., Begelman, M. C., 1999, Month.Not. R. A. Soc., 303, L1-L5

Blondin, J. M., Richards, M. T. \& Malinowski, M. L. 1995, ApJ, 445, 939

Carrol D. L., 1990, ApJ, 348, 588-596

Chakrabarti S.K., Theory of Transonic Astrophysical

Flows, World Scientific, Singapore, 1990

Chakrabarti, S. K. \& Molteni D. , 1993, ApJ, 417, 671

Chakrabarti, S. K., 1996, ApJ, 464, 664 
Chakrabarti, S. K., Acharyya, K., Molteni, D., 2004, A\&A, 421, 1

Chattopadhyay I., Das S., Chakrabarti, S. K., 2004, MNRAS, 348, 846-856

Cohen, J. G. \& Ryzhov, A., 1997, ApJ, 486, 230

Hachisu, Izumi, Kato, Mariko, Kato, Taichi, 2004, ApJ, 606, Issue 2, L139

Molteni, D., G. Lanzafame and S. Chakrabarti, 1994, ApJ , 425, 161

Miller M. C. , Lamb F. K., 1993, ApJL , 413, L43 - L46

Molteni, D., Sponholz, H., Chakrabarti, S. K., 1996, ApJ, 457, 805

Ryu, D., Chakrabarti, S.K. and Molteni, D., ApJ, 1997, 474,378

Molteni, D., Toth, G., Kuznetsov, O., 1999, ApJ, 516 , 411 Molteni, D., Kuznetsov, O. A., Bisikalo, D. V., Boyarchuk, A. A. 2001, MNRAS, 327, 1103-1012

Molteni D., Gerardi G., Valenza M. A., 2001,ApJL, 551, L77 - L80

Molteni, D., Acharya, K., Kuznetsov, O., Bisikalo, D., Chakrabarti, S. K. , 2001, ApJL, 563, L57

Paczyński, B., Wiita, P.J., 1980, A\&A, 88, 23

Sawada, K., Matsuda, T., Inoue, M., \& Hachisu, I. 1987, MNRAS, 224, 307

Smith, D. M., Heindl, W. A., Swank, J. H., 2002, ApJ, 569, 362

Spruit, H. C. 1987, A\&A, 184, 173-184

Spruit, H. C., Matsuda, T., Inoue, M., \& Sawada, K. 1987, MNRAS, 229, 517

Taam, R. E., Fu, A., \& Fryxell, B. A. 1991, ApJ, 371, 696

Yukawa, H., Boffin, H. M. J., \& Matsuda, T. 1997, MNRAS, 292, 321 